\title{
A novel envelope mediated post entry restriction of murine leukaemia virus in human cells is Ref1/ TRIM5 $\alpha$ independent
}

\author{
Nidia MM Oliveira ${ }^{\dagger}$, Roochi $_{\text {Trikha }}^{\dagger}$, Áine McKnight ${ }^{*}$
}

\begin{abstract}
Background: 'Intrinsic' resistance to retroviral infection was first recognised with the Friend virus susceptibility gene (FV1), which determines susceptibility to murine leukaemia virus (MLV) infection in different murine species. Similarly, the tripartite motif (TRIM) family of proteins determine lentiviral restriction in a primate host-species specific manner. For example rhesus TRIM5 $\alpha$ (rhTRIM5 $\alpha$ ) can potently restrict HIV-1 infection while human TRIM5 $\alpha$ (huTRIM5 $\alpha$ ) only has a mild effect on SIVmac and HIV-1 infectivity (LV1). Human TRIM5 $\alpha$ is able to restrict MLV-N virus replication, but is ineffective against MLV-B or MLV-NB virus infection. LV2 restriction of some HIV-2 viruses is seen in human cells. Like LV1, LV2 is a post-entry restriction factor, whose viral determinants have been mapped to the viral capsid (CA). Unlike LV1, however, LV2 is determined by envelope (Env) in addition to CA. Here we present evidence of a novel Env determined post entry restriction to infection in human cells of pseudotyped MLV-B and MLV-NB cores.
\end{abstract}

Results: We generated retroviral vectors pseudotyped with various gamma and lentiviral Envs on MLV-B and -NB CAs containing a green fluorescent protein (GFP) reporter. Flow cytometry was used to determine transduction efficiencies in NP2/CD4/CXCR4 (glioma cell line stably transduced with the HIV receptors) and HeLa/CD4 cell lines. The HeLa/CD4 cell line restricted both MLV CAs in an Env dependent manner, compared to NP2/CD4/CXCR4 cells. Quantitative polymerase chain reaction (QT-PCR) analysis of reverse transcription (RT) transcripts demonstrates that this restriction occurs at a post entry and RT level. siRNA knockdown of huTRIM5 $\alpha$ ruled out a direct role for this cellular component in mediating this restriction. We describe a previously unobserved Env determined restriction of MLV-B and MLV-NB CAs in HeLa/CD4 cells when pseudotyped with HIV-2 and RD114 Envs, but not gibbon ape leukaemia virus (GALV), HIV-1 or Amphotrophic (Ampho) Envs.

Conclusions: Our data further demonstrate the variability of Env and CA mediated susceptibility to post entry host cell restriction. We discuss the relevance of these findings in light of the growing evidence supporting the complexities involved in innate host immunity to retroviral infection.

\section{Background}

Retroviruses can cause a variety of diseases in their host species. Over-expression, integration near oncogenic loci, or the hosts' response to the proteins encoded by retroviral genes determine the type of disease manifested [1]. Greater understanding of host immunity against

\footnotetext{
* Correspondence: a.mcknight@qmul.ac.uk

† Contributed equally

HIV/AIDS Group, Centre for Immunology and Infectious Disease, Blizard Institute of Cell and Molecular Science, Barts and the London School of Medicine and Dentistry, 4 Newark Street, Whitechapel, London E1 2AT, UK
}

retroviruses is pertinent in the era of a global HIV/AIDS epidemic.

The murine leukaemia viruses (MLVs) are members of the gamma-retrovirus genus of the Retroviridae family. The diseases caused by MLVs include lymphomas and leukaemias. Studies on Friend MLV led to the discovery of the archetypal regulation or restriction of viral infection by intrinsic host-cell defence mechanisms. Friend virus susceptibility factor (Fv1) is a dominant allele expressed in mice or cell lines adapted from specific species of mice that confers resistance to different MLV strains [2]. Hence, MLV-N strains (N-tropic MLVs) are 
unable to infect mice with the $\mathrm{Fv}^{\mathrm{b} / \mathrm{b}}$ genotype, and MLV-B strains are unable to infect mice with the $\mathrm{Fv}^{\mathrm{n} / \mathrm{n}}$ genotype. Mice with the $\mathrm{Fv}^{\mathrm{n} / \mathrm{b}}$ genotype are resistant to both strains of MLVs but are susceptible to viruses that are both N and B tropic, such as Moloney MLV (MLVNB) [3]. Fv1 is a saturable gag-like element expressed from a murine endogenous retrovirus-L (MuERV-L) [4] closely related to the human ERV-L [5,6]. Fv1 blocks MLV virus prior to integration and does not block infection by other retroviruses [7]. On the virus side, historically, a single amino acid (aa) in the CA protein at position 110 is thought to determine if MLVs are B or $\mathrm{N}$ tropic $[8,9]$. However, more recent evidence suggests that residues up- or down- stream from this canonical site may also influence virus susceptibility to host immunity [10-12].

Less than a decade after Fv1 was cloned in 1996, the tripartite motif (TRIM) family of proteins were implicated in species-specific restriction of incoming retroviral CAs, initially referred to as the lentivirus restriction factor or Lv1 [13-15].

Unlike Fv1 restriction, the TRIM proteins have a broader reactivity and can act either before or after RT depending on the invading virus and host cell species. Rhesus TRIM5 $\alpha$ (rhTRIM5 $\alpha$ ) can potently restrict HIV1 infection; conversely human TRIM5 $\alpha$ (huTRIM5 $\alpha$ ) has only minor effects on SIVmac and HIV-1 infectivity. HuTRIM5 $\alpha$, however, can restrict MLV-N virus replication (Ref1), but not MLV-B or-NB virus infection $[14,16]$. Interestingly, mutations in the B30.2/SPRY domain of huTRIM5 $\alpha$ confer on it the ability to restrict MLV-B, SIVmac and HIV-2 viruses [10,17]. Yan and Kozak, [18] have described another CA mediated post entry resistance to the ecotropic MLV AKV in a murine cell line which is distinct from the classical Fv1 mediated restriction. This restriction is present in 3 out of the 4 major genera of Mus species, suggesting an extended role of Fv1 in Mus evolution and retroviral resistance pre-dating the classical Fv1 alleles determined for laboratory mice [19].

Evidence for Env mediated post entry restriction is gaining momentum. Previously, we described another lentivirus susceptibility factor termed Lv2; like Lv1 and Ref1, Lv2 is a post-entry restriction found in some human cells and their derivatives. However, in addition to $\mathrm{CA}$, the virus Env also has a role in Lv2 restriction. The molecular clone non-restricted $(\mathrm{MCN})$ virus derived from a tissue culture adapted HIV-2 isolate has a nonrestricted phenotype, while the molecular clone restricted (MCR) virus derived from a primary isolate from the same patient is susceptible to Lv2. The Env mediated restriction of MCR is overcome by pseudotyping viral cores with VSV-G Env. Envelope and CA substitutions between MCR and MCN retroviral vectors pinpointed aa 74 in the Env and aa 207 in the CA as the viral determinants for Lv2 MCR restriction [20,21]. Another post-entry HIV-1 resistance factor, termed Lv3 [22], has been described in rhesus macaques and is dependent on CD4 and endogenous co-receptor viral delivery. Uchil et al. [23] have pseudotyped the avian leukaemia virus-A (ALV-A) Env onto HIV-1, MLV-N, and MLV-B cores and described novel effects on post viral entry in HEK293 cells transiently transfected with different murine and human TRIMs. Together, these studies suggest that the Env protein plays a role in mediating events post entry, at or during reverse transcription.

Given such subtleties in Env and CA determinants of intrinsic host cell resistance, we wished to determine if the Lv2 phenotype was operative for the MLVs in human cells. Because the huTRIM5 $\alpha$ restriction of VSV-G Env pseudotyped MLV-N viruses is well characterised, we sought to determine the role, if any, of other human host restriction factors on the MLV-B and MLV-NB CAs. We tested our hypothesis by pseudotyping these CAs with two HIV-2 Envs that defined the Lv2 restriction in HeLa/CD4 cells.

This paper describes the restriction of MLV-B and MLV-NB viral cores in human cells when pseudotyped with HIV-2 $[20,21]$ and the feline endogenous virus RD114 Envs. In comparison the same cores pseudotyped by the gamma-retroviral gibbon ape leukaemia virus (GALV), Amphotrophic (Ampho) MLV and the VSV-G Envs were relatively unrestricted in $\mathrm{HeLa} / \mathrm{CD} 4$ cells.

Thus we provide evidence for a novel restriction of MLV-B and MLV-NB viruses in the human HeLa/CD4 cell line, which is dependent on the pseudotyping Env. This restriction is distinct from the previously characterised Ref1, Lv1, Lv2 and Lv3. Our data add another layer to the intricate puzzle of the relationships between different retroviruses and the myriad of host cell defences they encounter.

\section{Results}

HIV-2 Envs MCN and MCR mediate MLV-B and MLV-NB restriction in HeLa/CD4 cells which is rescued by different retroviral Envs

Previously we had demonstrated that an Env and a CA derived from an HIV-2 isolate, MCR, were determinants of Lv2 restriction in HeLa/CD4 cells. In contrast, the Env and CA derived from the MCN isolate are relatively unrestricted in the same cells. The susceptibility of MLV-N CA to Lv1 in human cells is well documented $[14,16,24]$. Here we sought to determine if MLV-B and MLV-NB cores would be restricted in an Lv2-like manner. However, unexpectedly, we observed that if MLV-B cores are pseudotyped with either the MCN or MCR HIV-2 Envs they both result in a restricted phenotype in 
HeLa/CD4 cells, but not in the NP2/CD4/CXCR4 (a permissive glioma cell line stably transduced with the HIV receptors [25]) cells (Figure 1a). Infection on NP2/ CD4/CXCR4 is 60X (MCN) and 200X (MCR) greater than on the restricted HeLa/CD4 cells (Figure 1b). In contrast, the VSV-G and Ampho pseudotyped MLV-B vectors were relatively uninhibited in both cell lines, showing only $2 \mathrm{X}$ and $5 \mathrm{X}$ differences in infection (Figures $1 \mathrm{a}$ and $1 \mathrm{~b})$. Similar results were obtained for the
MLV-NB CA pseudotyped with these Envs (Figure 1a). Infection of NP2/CD4/CXCR4 cells is 35X (MCN) and 160X (MCR) greater than on HeLa/CD4 cells. The VSV-G and Ampho pseudotyped MLV-NB vectors remain unrestricted, showing only $1 \mathrm{X}$ and $5 \mathrm{X}$ differences in infection of the two cell lines (Figure 1b). Thus we have shown that this restriction of MLV-B and -NB CAs is Env dependent. To further confirm that this restriction is also dependent on viral CA, we produced

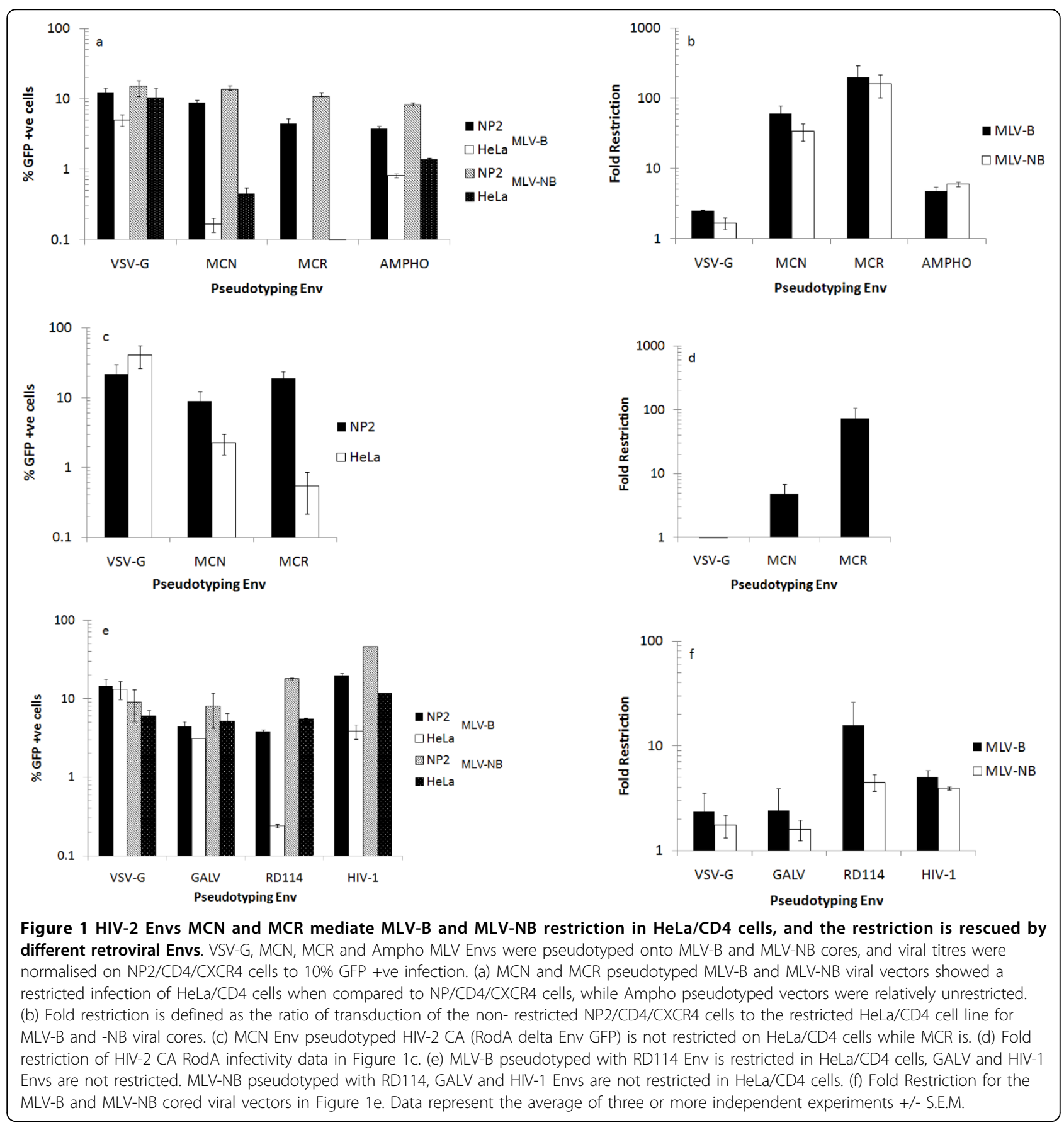


retroviral vectors with an unrelated HIV-2 CA, RodA. As expected, when we pseudotyped the RodA delta Env HIV-2 core with VSV-G, MCN and MCR Envs only the MCR Env was restricted in HeLa/CD4 cells (Figure 1c). Specifically, the VSV-G and the MCN pseudotyped vectors were unrestricted $(0.6 \mathrm{X}$ and $4.7 \mathrm{X}$ difference between NP2 and HeLa cells), while the MCR Env showed 73X difference in infection (Figure 1d) similar to the Lv2 phenotype described previously [20]. Hence the restriction with MLV-B and MLV-NB is similar to Lv2 in its HeLa/CD4 specificity and is overcome by pseudotyping with VSV-G Env. Unlike Lv2, however, both the MCN and MCR Envs result in a restricted phenotype with these viral CAs. Importantly, these results show that HIV-2 Envs reveal a previously unreported restriction of MLV-B and MLV-NB cores in HeLa/CD4 cells.

Next we generated retroviral vectors with HIV-1 [26], GALV [27,28] and RD114 Envs [29]. These Envs were chosen to expand the receptor classes of these MLV CA for entry into human cells and have been well characterised as pseudotyping Envs for MLV cores [29,30]. GALV Env receptor, PiT1 [31] is closely related to the Ampho Env receptor PiT2 [32,33] and HIV-1 uses the same receptors as the HIV-2 Envs. Both MLV-B and MLV-NB pseudotyped viral cores were rescued from the restriction on HeLa/CD4 cells by the GALV and HIV-1 Envs (Figure 1e). The $2.4 \mathrm{X}$ and $5 \mathrm{X}$ difference in infection for GALV and HIV-1 are comparable to the VSV-G control (2.3X) (Figure 1f). Similarly, GALV (1.6X), HIV-1 (4X) and RD114 (4.5X) pseudotyped MLV-NB CAs were unrestricted (Figures 1e and 1f). Interestingly, the RD114 Env (which uses the neutral amino acid transporter abundantly expressed in human cells [29]) showed the highest levels of restriction compared to the VSV-G Env on the MLV-B CA (Figure 1e), with a 16X difference in infection of the two cell lines (Figure 1f). However, this level of restriction is less than that conferred by the HIV-2 Envs described above.

\section{Flow cytometry analysis demonstrates that NP2/CD4/ CXCR4 and HeLa/CD4 cells express similar levels of receptors on their cell surface}

The results above demonstrate that Env is a strong determinant of the ability of viral core to complete early events in replication. We next determined the HIV receptor levels of NP2 and HeLa cells to further support the notion that the restriction was not due to a difference in the expression of these receptors. We immunostained the cells with fluorescently labelled antibodies to CD4 and CXCR4 and used flow cytometry for their detection. These experiments show similar log shifts in fluorescent intensity for CD4 staining (Figure 2a) and CXCR4 staining (Figure 2b) on both HeLa/CD4 and NP2/CD4/CXCR4 cells. Hence, the restriction in HeLa/ CD4 cells is not due to the reduced level of receptors

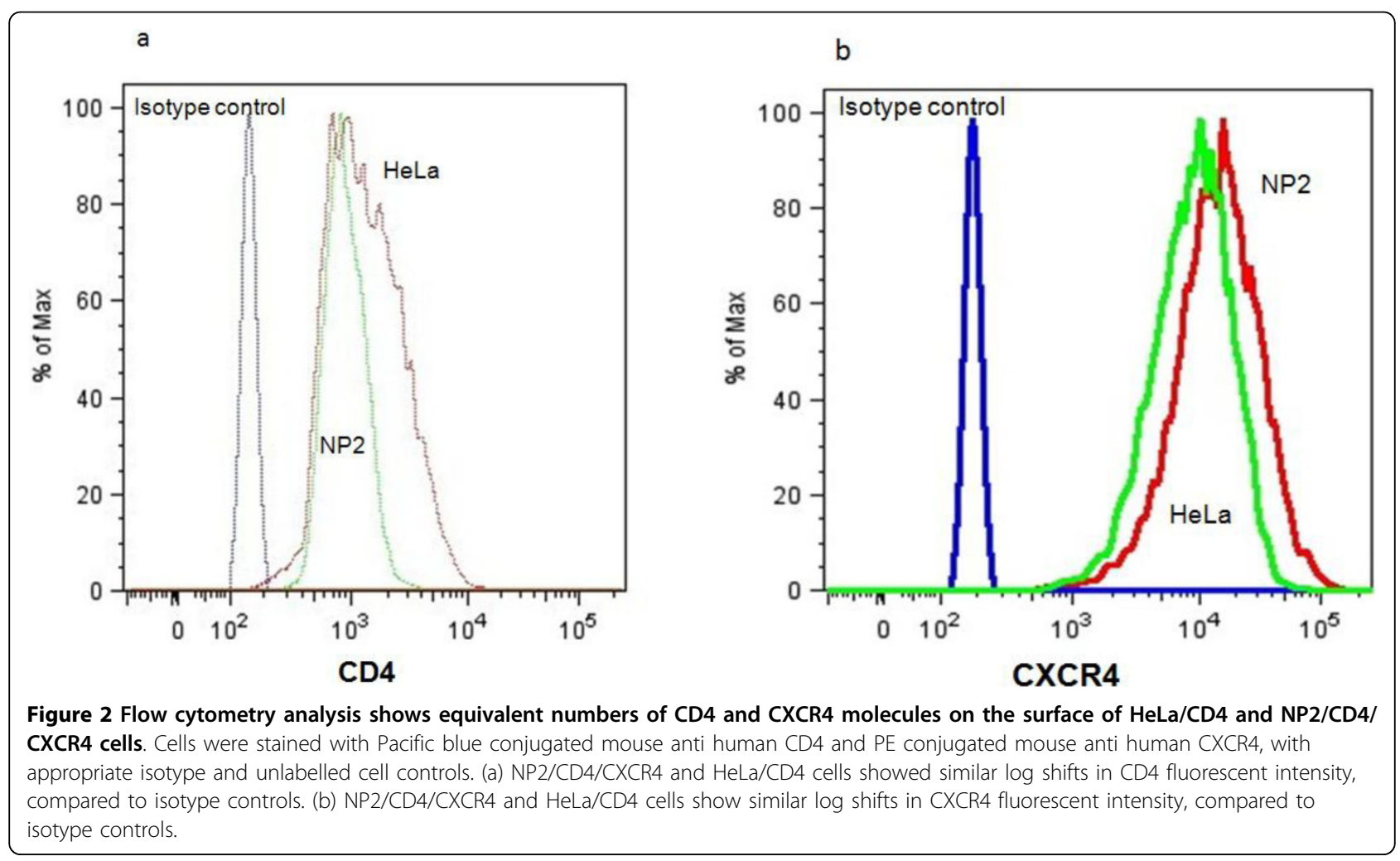


on the surface of these cells in comparison to those seen on the unrestricted NP2/CD4/CXCR4 cells.

\section{QT-PCR data demonstrate that the MLV-B and MLV-NB restriction in HeLa/CD4 cells is post entry}

We used QT-PCR to monitor retroviral transcription initiated after viral and plasma membrane fusion. HeLa/CD4 and NP2/CD4/CXCR4 cells were challenged with different pseudotyped viruses, at MOIs of 0.25 to 1 , and incubated for $16 \mathrm{hrs}$ or more to allow RT to proceed. Negative controls for infection included incubating virus for 5 minutes at $4^{\circ} \mathrm{C}$ or adding trypsin to target cells just prior to challenge (to strip the cell surface expression of receptors). In all cases viral inocula were DNase treated with 100 units DNase/ml of virus for $11 / 2 \mathrm{hrs} 37^{\circ} \mathrm{C}$ and trypsinised off cells before lysis for DNA extraction. The extracted DNA was assessed by QT-PCR for late RT products. Primers and probes were designed to amplify the transfer plasmid pCNCG with an internal GAPDH control reaction. All data were normalized to the genomic GAPDH internal control (GAPDH amplification results are not shown).

Figure 3a (i) shows that the level of newly transcribed DNA transcripts after $\mathrm{O} / \mathrm{N}$ incubation $37^{\circ} \mathrm{C}$ on $\mathrm{HeLa} /$ CD4 cells were equivalent (MOI 1). The corresponding infectivity data (Figure 3a (ii)) show that the MCN pseudotyped virus is, however, much less infectious compared to VSV-G Env pseudotyped virus. Figure 3b (i and ii) shows that regardless of viral Env, pseudotype infection of NP2 cells always resulted in greater numbers of RT transcripts compared to infection of HeLa cells. Importantly, however, a comparison of transcripts produced after challenge of HeLa cells resulted in the same number of transcripts regardless of whether the CA was pseudotyped with restrictive or non-restrictive Envs. The levels of RT transcripts detected correspond to the different MOIs used to challenge cells. The corresponding infectivity data (Figure $3 \mathrm{~b}$ (iii and iv)) confirm that in NP2 cells the transcripts result in permissive titratable infection for both Envs, but in HeLa cells, while the HIV-1 Env virus results in efficient infection, the HIV-2 MCN virus is restricted.

Similar results were observed with the different pseudotyped combinations described in this paper. In Figure 3c we show that the GALV and Ampho Envs produce similar levels of transcripts in HeLa cells.

In summary, the data showed that viral transcripts were overall reduced in HeLa cells when compared to NP2 cells, in a dose dependent manner. Importantly, these QT-PCR data show that the number of RT transcripts for the restricted Envs are similar to the nonrestricted Envs in HeLa cells. These data thus confirm that the restriction seen in HeLa cells is not due to a block of viral entry at the plasma membrane, but is at a post entry post RT level.

\section{The observed restriction to MLV-B and MLV-NB in HeLa/ CD4 cells is not mediated by huTRIM5 $\alpha$}

HuTRIM5 $\alpha$ has been well documented as a restriction to MLV-N, but not MLV-B and MLV-NB pseudotyped with a VSV-G Env. We down regulated TRIM5 $\alpha$ production using specific siRNA knockdown to determine whether or not it had a role in the restricted phenotype described here. As shown by others, siRNA knockdown of huTRIM $5 \alpha$ relieved the restriction in HeLa/CD 4 (with an increase from $18 \%$ to $38 \%$ in transduction) of VSV-G pseudotyped MLV-N viral vectors, but not with control siRNA (Figure 4a). Similar results were obtained on NP2/CD4/CXCR4 cells (7.6\% to $26.4 \%$ (data not shown). By comparison, siRNA knockdown of huTRIM5 $\alpha$ did not affect the HIV-2 (MCN, MCR) and RD114 Env mediated restriction of MLV-B CAs in the $\mathrm{HeLa} / \mathrm{CD} 4$ cells (Figure 4a). The results were similar for MLV-NB cored viruses (Figure $4 \mathrm{~b}$ ). Therefore, the restriction of MLV-B and MLV-NB cores, pseudotyped by HIV-2 and RD114 Envs, is not due to the direct activity of huTRIM $5 \alpha$ on incoming CAs.

\section{Discussion}

Here we describe a novel post entry restriction in HeLa/ CD4 cells of MLV-B and MLV-NB viral cores when pseudotyped with two HIV-2 Envs and one RD114 Env, demonstrating that human cells have factors other than TRIM5 $\alpha$ which inhibit MLV infection.

Studies of the post-entry actions of host cellular factors to block specific retroviral CAs from establishing active infections have provided a greater understanding of different retroviral/host interactions and their co-evolution. These findings imply that retroviruses evolve envelopes to avoid innate host defences that target retroviral infections.

While the study of innate retroviral host cell immunity to incoming CAs has typically employed VSV-G Env pseudotyped vectors, there is a growing body of evidence to suggest that the viral entry route also contributes to the outcome of infection [17,20-22]. Previously, we demonstrated the Lv2 restriction in HeLa/CD4 cells to a molecular clone of an HIV-2 isolate, MCR. Unlike Lv1 and Fv1, Lv2 is determined by Env in addition to CA. Lv2 restriction is overcome by the substitution of MCR Env/CA with either their non-restricted MCN equivalents or with VSV-G Env [20,21]. Similarly the Minr resistance factor [18] has recently been shown in cells from the African pygmy mouse $M$. minutoides against non lab-adapted strains of AKV ecotropic MLVs. Minr also acts post viral entry, and is distinct from any of the Fv1 allelic restrictions described 
previously. It was noted that lab-adapted MoMLV and FRMLV57 Envs displayed a 10- fold greater titre than AKV Envs in NIH3T3 cells when compared to M. minutoides cells, and speculated that like Lv2 there may be an Env dependent pre-RT determinant of Minr resistance [18].
Given the extent to which MLV-B, MLV-NB and MLV-N CAs have been investigated and their differences in susceptibility to Fv1 and huTRIM5 $\alpha$ restriction $[9-12,16,17,23]$, we specifically determined whether or not the delivery of MLV-B and -NB CAs into human cells by the MCN and MCR HIV-2 Envs would affect 


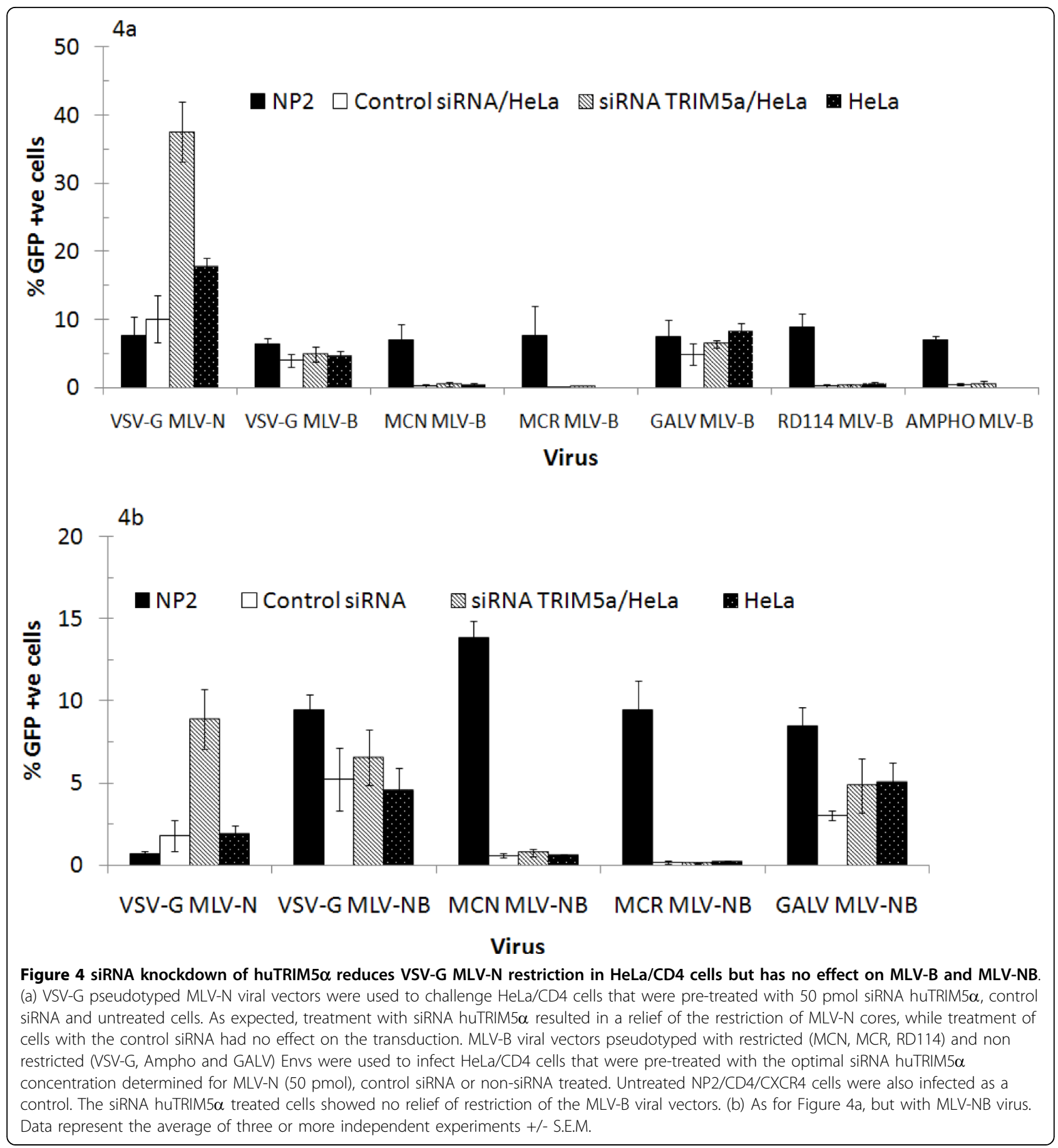

transduction in a manner consistent with the Lv2 restriction.

Surprisingly, our data revealed a restriction distinct from Lv-2 because both MLV-B and MLV-NB cored viruses were restricted in $\mathrm{HeLa} / \mathrm{CD} 4$ cells if pseudotyped by either HIV-2 Env. GALV and VSV-G Envs rescued this restriction. By comparison, the RD114 Env pseudotyped MLV-B virus was restricted, but not to the levels seen with the HIV-2 Envs. It is interesting that the MLV-NB core was not restricted if delivered by an RD114 Env. This difference may reflect the adaptive changes MLV-NB has undergone in relation to both MLV-N and MLV-B typified by its lack of sensitivity to both Fv1 and huTRIM5 $\alpha$ activity $[9-12,16,17,23]$.

Ampho Env pseudotyped MLV-B and MLV-NB cores were relatively more restricted than GALV and less 
restricted than RD114 Envs (Figures 1a, b, e and 1f). It is well documented that GALV Env pseudotyped retroviral vectors show higher transduction efficiencies than Ampho Env vectors on human cells [34]. The prevailing hypothesis is that the discrepancy in target cell receptor density levels of PiT1 [31] (GALV receptor) and PiT2 (Ampho receptor) contribute to these differences $[33,35]$. However, we show by QT-PCR that there is no difference in the levels of late RT transcripts between GALV and Ampho Env vectors in HeLa/CD4 cells (Figure 3c). Hence the difference in susceptibility is unlikely to be entirely due to just receptor density levels and perhaps reflects subtleties in the adaptation to different host species.

Both HIV-1 and HIV-2 use CD4 and CXCR4 as receptors for entry. Unlike the HIV-2 Envs, the HIV-1 Env pseudotyping did not restrict MLV CAs in HeLa/CD4 cells. Fluorescent receptor labelling with detection by flow cytometry revealed similar amounts of both molecules on the surface of both HeLa/CD4 and NP2/CD4/ CXCR4 cells (Figures 2a and 2b). These data suggest that the restriction we describe is not due to a lack of entry because of differences in receptor density. Furthermore, the QT-PCR data demonstrates that the levels of RT transcripts for the restricted HIV-2 MCN and nonrestricted HIV-1 pseudotyped viruses were similar (Figures $3 \mathrm{~b}$ ( $\mathrm{i}$ and ii)).This conclusion is further supported by the QT-PCR data which showed that the levels of transcripts in the HeLa/CD4 cells were similar for the different restricted and non restricted Envs (Figures 3a-c). These data combined suggest a post entry, post RT mechanism of action involved in the HeLa/CD4 cells.

Given the data presented here and the increasing evidence that host cell restriction is not solely dependent on the susceptibility of an incoming viral CA; a central question remains one of why/how? HIV-1 has rapidly adapted from its SIV progenitor and led to a world-wide HIV epidemic, whereas the closely related HIV-2 has not. Previous studies have shown that HIV-2 replication in primary macrophages is characterised by what is believed to be a 'latent state' when compared to continuous HIV-1 production [36]. It is possible that the adaptations of HIV-1 Env are more successful than those of HIV-2, with HIV-1 adapting its route of entry to override some of the innate human defences it has hitherto encountered.

Since MLV-N is restricted by huTRIM5 $\alpha$, we used siRNA knockdown experiments to determine if huTRIM5 $\alpha$ had a role in this novel restriction of MLV$B$ and MLV-NB CAs. Our data clearly demonstrated that while the siRNA huTRIM5 $\alpha$ treatment relieved restriction of VSV-G pseudotyped MLV-N virus, this was not the case with MLV-B and MLV-NB cores (Figures $4 \mathrm{a}$ and $4 \mathrm{~b})$.
In summary, our data indicate that there is a novel post entry restriction to MLV-B and -NB CAs in human cells and provide more evidence on Env contribution of CA restriction by innate retroviral responses.

\section{Methods \\ Cell lines}

HeLa/CD4 (human squamous epithelial carcinoma) cells and human glioma cell NP2/CD4/CXCR4 [25] were maintained in Dulbecco's modified essential medium (DMEM) supplemented with $10 \%$ foetal calf serum (FCS), $60 \mu \mathrm{g}$ of penicillin/ml, $100 \mu \mathrm{g}$ of streptomycin/ $\mathrm{ml}$, and $1 \mathrm{mg}$ of G418/ml. NP2/CD4/CXCR4 DMEM also contained $1 \mu \mathrm{g}$ of puromycin/ml. Mus dunni tail fibroblasts (MDTF) and $293 \mathrm{~T}$ cells were maintained in DMEM supplemented with 10\% FCS, with the penicillin and streptomycin concentrations as described above.

\section{Expression Plasmids}

The mammalian expression plasmids used to generate retroviral vectors in this study included: pMDG VSV-G Env; pMP11-MCRenv, pMP11-sMCNenv [20], GALV Env [37], Ampho Env [38], RD114 Env, pCIG3-N MLV$\mathrm{N}$ core, pCIG3-B MLV-B core, pCIG3 MLV-NB core, pCNCG transfer plasmid encoding enhanced GFP (eGFP) [39].

\section{Production of retroviral vectors}

Retroviral vectors were produced using a three plasmid transfection system in $293 \mathrm{~T}$ cells as described previously [39]. $293 \mathrm{~T}$ cells were passaged $24 \mathrm{hrs}$ prior to transfection and seeded at $2.5-3 \times 10^{6}$ cells in a $10-\mathrm{cm}^{2}$ tissue culture dish. The polyethylenimine (PEI, Sigma) transfection reagent was used in all transfections (stock $1 \mathrm{mg} / \mathrm{ml}$ ). Plasmids were transfected at a ratio of 1:2:3 Env:Core:Transfer. A total of $18 \mu \mathrm{g}$ DNA was combined with $60 \mu \mathrm{l}$ of $1 \mathrm{mg} / \mathrm{ml} \mathrm{PEI}$ in $900 \mu \mathrm{l}$ of serum-free DMEM (Gibco, Invitrogen Corp.). The suspension was vortexed briefly, incubated for $10 \mathrm{mins}$ at room temperature and added drop-wise to the $293 \mathrm{~T}$ cells. Transfected cells were incubated for a minimum of $5 \mathrm{hrs}$ or $\mathrm{O} / \mathrm{N}$ at $37^{\circ} \mathrm{C}(5 \%$ [vol/vol $\left.] \mathrm{CO}_{2}\right)$ in a humidified incubator; transfection supernatant was removed and cells were overlaid with $10 \mathrm{ml}$ of fresh 10\% FCS DMEM for 48 and 72 hrs harvests of viral vector supernatants.

Transfection supernatants were filtered through a 0.45 $\mu \mathrm{m}$-pore-size filter (Millipore, Bedford MA, USA) and viral vector-containing medium stored in $500 \mu \mathrm{l}$ aliquots at $-80^{\circ} \mathrm{C}$ until required.

\section{Infection assays}

Infection assays of different cell lines were carried out by seeding cells at a density of $2 \times 10^{4}$ cells/well in 48 well cell culture plates in a total volume of $500 \mu \mathrm{l}$ 
growth DMEM, one day prior to challenge with the different pseudotyped retroviral vectors. The next day viral vectors were applied to the cells, in a total volume of $200 \mu \mathrm{l} /$ well (supplemented with 5\% FCS DMEM). The medium was removed 2-3 hrs later and replaced with fresh DMEM and the cells were cultured for a further $48 \mathrm{hrs}$ before analysis for the expression of GFP positive cells by flow cytometry with a FACScan (Becton Dickin-

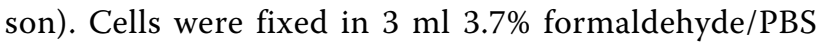
for 3 mins at RT, pelleted by centrifugation at 1500 $\mathrm{RPM}$, washed once in $1 \times \mathrm{PBS}$ and resuspended in $200 \mu \mathrm{l}$ PBS for flow cytometry. MLV-B and MLV-NB cored virus stocks were titrated and normalised on NP2/CD4/ CXCR4 cells and MLV-N virus stocks on MDTF cells before their addition to the target cell lines. Fold restriction ( $\mathrm{x}$-fold) is calculated as the proportion of GFP +ve cells in a non-restricted infection/restricted infection.

\section{siRNA knockdown of huTRIM5 $\alpha$ Optimisation of HiperFect: siRNA concentrations}

$\mathrm{HeLa} / \mathrm{CD} 4$ cells were seeded in a 6 -well plate at a density of $2 \times 10^{5}$ cells/well in a total volume of $2 \mathrm{ml}$, one day prior to transfection. HuTRIM $5 \alpha$ and a scrambled control siRNA were titrated with varying volumes of HiPerFect transfection reagent as per manufacturer's guidelines (QIAGEN, UK). In short, HiPerfect and siRNAs were individually diluted in serum free OPTIMEM, then mixed and incubated for $10 \mathrm{~min}$ RT. siRNA transfection mixtures were added drop wise to HeLa/CD4 cells and incubated in a total of $1 \mathrm{ml}$ serum free OPTIMEM for $30 \mathrm{mins}$ at $37^{\circ} \mathrm{C}$ in a humidified incubator. Cells were overlaid with $1.5 \mathrm{ml}$ fresh $10 \%$ FCS DMEM and incubated $\mathrm{O} / \mathrm{N}$. Non siRNA treated HeLa/CD4 cells were included as controls. The next day, siRNA transfection supernatants were removed and cells were challenged with VSV-G pseudotyped MLV-N viral vectors. Infected cells were incubated for a further $48 \mathrm{hrs}$ before flow cytometry analysis for the expression of GFP.

$\mathrm{HeLa} / \mathrm{CD} 4$ cells were seeded in a 48 -well plate at a density of $2 \times 10^{4}$ cells/well in a total of $500 \mu \mathrm{l}$, one day prior to transfection. Optimised HiPerFect and siRNA huTRIM5 $\alpha$ and a control siRNA were transfected as above and the next day transfected cells were infected with equal amounts of MLV-B or MLV-NB viral vectors pseudotyped with the different Env (VSV-G Env MLV-N control) which were previously normalized on NP2/CD4/ CXCR4 cells. NP2/CD4/CXCR4 cells were infected with equal amounts of virus as a positive control for viral infectivity. Infected cells were incubated for a further $48 \mathrm{hrs}$ before flow cytometry analysis for the expression of GFP.

\section{Detection of CD4 and CXCR4 expression on HeLa/CD4 and NP2/CD4/CXCR4 cells}

$\mathrm{HeLa} / \mathrm{CD} 4$ and NP2/CD4/CXCR4 cells were seeded in 6 well plates at a density of $5 \times 10^{5}$ cells/well in a total of
$2 \mathrm{ml}$ DMEM culture medium. The following day, cells were removed from the plate with $5 \mathrm{mM}$ EDTA, pelleted and incubated with $5 \mu \mathrm{l} / 100 \mu \mathrm{l}$ PBS Pacific blue conjugated mouse anti human $\mathrm{CD} 4$ and PE conjugated mouse anti human CXCR4. Mouse anti human IgG isotype controls were used for each antibody and unlabelled cells were also included as controls. Cells were stained at RT for $1 \mathrm{hr}$ then washed and fixed for flow cytometry analysis.

\section{Quantitative late PCR}

HeLa/CD4 and NP2/CD4/CXCR4 cells were seeded in a 12 -well plate at a density of $8 \times 10^{4}$ cells/well in a total volume of $1 \mathrm{ml}$, one day prior to infection. The following day, the spent medium was replaced with up to $1 \mathrm{ml}$ of fresh medium containing $4 \times 10^{4}-1.6 \times 10^{5} \mathrm{IUs} / \mathrm{ml}$ (MOI of $0.25-1$ ) of viral vectors. All vector stocks were pre-treated with $100 \mathrm{U}$ of $\mathrm{DNaseI} / \mathrm{ml}$ virus for $1 \mathrm{hr} 30 \mathrm{mins}$ at $37^{\circ}$ $\mathrm{C}([40])$ to degrade excess plasmid DNA from the transfection stage. Normal infections were incubated $\mathrm{O} / \mathrm{N} 37^{\circ} \mathrm{C}$. Controls for infection included trypsinising the cells just prior to infection in 10× Trypsin/EDTA for 15 minutes at $37^{\circ} \mathrm{C}$ (to strip off the cell surface receptor expression) or absorbing virus at $4^{\circ} \mathrm{C}$ for 5 minutes with immediate lysis. All virus incocula were trypsinised off infected cells after the appropriate incubation times and temperatures, followed by $2 \times$ washes in $10 \times$ Trypsin/EDTA, before lysis and DNA extraction with a QIAamp blood DNA mini kit according to the manufacturer's protocol (QIAGEN, UK).

The isolated DNA was subjected to quantitative PCR (QT-PCR) to determine the late GFP RT transcripts present. Each $25 \mu \mathrm{l}$ reaction mixture for the detection of late GFP RT DNA transcripts contained the following components: $1 \times$ MegaMix PCR buffer (Microzone Limited), 400 nM forward primer (5'-CAACAGCCACAACGTCTATATCAT-3'), 400 nM reverse primer (5'-ATGTTGTGGCGGATCTTGAAG-3'), 100 nM probe (5'-6carboxyfluorescein- CCGACAAGCAGAAGAACGGCATCAA -6- carboxy-tetrafluorescein-3'), and $500 \mathrm{ng}$ of total DNA. As a control for the total amount of DNA used in each reaction, GAPDH forward and reverse primers and a CY5 probe were also included in each sample tested. A standard curve was prepared with the pCNCG transfer plasmid in a background of $200 \mathrm{ng}$ of salmon sperm carrier genomic DNA (data not shown).

PCR amplifications, data acquisition, and analysis were performed with the ABI PRISM 7500 sequence detection system. PCR conditions consisted of one cycle of denaturation $\left(95^{\circ} \mathrm{C}\right.$ for $5 \mathrm{~min}$ ) followed by 40 cycles of amplification $\left(95^{\circ} \mathrm{C}\right.$ for $15 \mathrm{~s}, 60^{\circ} \mathrm{C}$ for $\left.1 \mathrm{~min}\right)$.

\section{Abbreviations}

MLV: murine leukaemia virus; HIV-1 and 2: human immunodeficiency virus types 1 and 2; VSV-G: vesicular stomatitis virus G protein; Fv1: Friend virus 
susceptibility gene 1; Ref1: retrovirus restriction factor 1; LV1-3: lentivirus restriction factors 1-3; TRIM: tripartite motif; Env: envelope; CA: capsid; RT: reverse transcription; QT-PCR: quantitative PCR; siRNA: small interfering RNA; MCR: molecular clone restricted; MCN: molecular clone non restricted; RD114: feline endogenous retrovirus; GALV: gibbon ape leukaemia virus; Ampho: Amphotrophic; aa: amino acid; MDTF: Mus dunni tail fibroblasts; GFP: green fluorescent protein; MOl: multiplicity of infection.

\section{Acknowledgements}

We would like to acknowledge Massimo Pizzato (Imperial College, London) for the pEnvHXBDCT (HIV-1 Env in the text). We would like to thank David Marchant for the initial experiments, Kelly Cheney, Matthias Dittmar, Hanna Dreja, lan Harrison and Li Liu for reading the manuscript and useful discussions. This work was funded by the Medical Research Council UK (Non-Clinical Senior Fellowship G117/547, Áine McKnight).

\section{Authors' contributions}

NMMO designed and carried out experiments and drafted the manuscript. RT carried out experiments and drafted the manuscript. AM conceived the study and participated in experimental design and co-ordination and helped draft the manuscript. All authors read and approved the final manuscript prior to submission.

\section{Competing interests}

The authors declare that they have no competing interests.

Received: 12 November 2009 Accepted: 7 October 2010 Published: 7 October 2010

\section{References}

1. Coffin J: Retroviridae: the viruses and their replication. In Field's Virology. Edited by: Fields B, Knipe DM, Howley PM. Philadelphia, Pa.: Lippincott; , Third 1996:2:1767-1848

2. Coffin JM: Retrovirus restriction revealed. Nature 1996, 382:762-763.

3. Tennant RW, Schluter B, Yang W, Brown A: Reciprocal inhibition of mouse leukemia virus infection by Fv-1 allele cell extracts. Proc Natl Acad Sci USA 1974, 71:4241-4245.

4. Benit L, De Parseval N, Casella JF, Callebaut I, Cordonnier A, Heidmann T: Cloning of a new murine endogenous retrovirus, MuERV-L, with strong similarity to the human HERV-L element and with a gag coding sequence closely related to the Fv1 restriction gene. J Virol 1997 71:5652-5657.

5. Best $\mathrm{S}$, Le Tissier P, Towers G, Stoye JP: Positional cloning of the mouse retrovirus restriction gene Fv1. Nature 1996, 382:826-829.

6. Cordonnier A, Casella JF, Heidmann T: Isolation of novel human endogenous retrovirus-like elements with foamy virus-related pol sequence. J Virol 1995, 69:5890-5897.

7. Goff SP: Operating under a Gag order: a block against incoming virus by the Fv1 gene. Cell 1996, 86:691-693.

8. DesGroseillers L, Jolicoeur P: Physical mapping of the Fv-1 tropism host range determinant of BALB/C murine leukemia viruses. J Virol 1983, 48:685-696.

9. Kozak CA, Chakraborti A: Single amino acid changes in the murine leukemia virus capsid protein gene define the target of Fv1 resistance. Virology 1996, 225:300-305.

10. Maillard PV, Reynard S, Serhan F, Turelli P, Trono D: Interfering residues narrow the spectrum of MLV restriction by human TRIM5alpha. PLOS Pathog 2007, 3:e200.

11. Lassaux A, Sitbon M, Battini $J$ : Residues in the murine leukemia virus capsid that differentially govern resistance to mouse Fv1 and human Ref1 restrictions. J Virol 2005, 79:6560-6564.

12. Ulm JW, Perron M, Sodroski J, R CM: Complex determinants within the Moloney murine leukemia virus capsid modulate susceptibility of the virus to Fv1 and Ref1-mediated restriction. Virology 2007, 363:245-255.

13. Stremlau M, Owens CM, Perron MJ, Kiessling M, Autissier P, Sodroski J: The cytoplasmic body component TRIM5alpha restricts HIV-1 infection in Old World monkeys. Nature 2004, 427:848-853.

14. Goff SP: Retrovirus restriction factors. Mol Cell 2004, 16:849-859.

15. Towers GJ, Goff SP: Post-entry restriction of retroviral infections. AIDS ReV 2003, 5:156-164.
16. Yap MW, Nisole S, Lynch C, Stoye JP: Trim5alpha protein restricts both HIV-1 and murine leukemia virus. Proc Natl Acad Sci USA 2004, 101:10786-10791.

17. Diaz-Griffero F, Perron M, McGee-Estrada K, Hanna R, Maillard PV, Trono D, Sodroski J: A human TRIM5alpha B30.2/SPRY domain mutant gains the ability to restrict and prematurely uncoat B-tropic murine leukemia virus. Virology 2008, 378:233-242.

18. Yan Y, Kozak CA: Novel post-entry resistance to AKV ecotropic mouse gammaretroviruses in the African pygmy mouse, Mus minutoides. J Virol 2008.

19. Yan Y, Buckler-White A, Wollenberg K, Kozak CA: Origin, antiviral function and evidence for positive selection of the gammaretrovirus restriction gene Fv1 in the genus Mus. Proc Natl Acad Sci USA 2009, 106:3259-3263.

20. Schmitz C, Marchant D, Neil SJ, Aubin K, Reuter S, Dittmar MT, McKnight A: LV2, a novel postentry restriction, is mediated by both capsid and envelope. J Virol 2004, 78:2006-2016.

21. Reuter $S$, Kaumanns $P$, Buschhorn SB, Dittmar MT: Role of HIV-2 envelope in LV2-mediated restriction. Virology 2005, 332:347-358.

22. Pineda MJ, Orton BR, Overbaugh J: A TRIM5alpha-independent post-entry restriction to HIV-1 infection of macaque cells that is dependent on the path of entry. Virology 2007, 363:310-318.

23. Uchil PD, Quinlan BD, Chan WT, Luna JM, Mothes W: TRIM E3 ligases interfere with early and late stages of the retroviral life cycle. PLOS Pathog 2008, 4:e16.

24. Nisole S, Stoye JP, Saib A: TRIM family proteins: retroviral restriction and antiviral defence. Nat Rev Microbiol 2005, 3:799-808.

25. Soda Y, Shimizu N, Jinno A, Liu HY, Kanbe K, Kitamura T, Hoshino H Establishment of a new system for determination of coreceptor usages of HIV based on the human glioma NP-2 cell line. Biochem Biophys Res Commun 1999, 258:313-321.

26. Reil H, Bukovsky AA, Gelderblom HR, Gottlinger HG: Efficient HIV-1 replication can occur in the absence of the viral matrix protein. EMBO 1998, 17:2699-2708.

27. Kawakami* TG, Buckley P, Huff S: Characterization of a C-type virus associated with gibbon ape lymphosarcoma. Med Primat 1972, 163-168, Proc. Third Conf. Exp. Surg Primates Lyon 1972 part III:

28. Eglitis MA, Schneiderman RD, Rice PM, Eiden MV: Evaluation of retroviral vectors based on gibbon ape leukamia virus. Gene Therapy 1995, 2:486-492

29. Lucas ML, Seidel NE, Porada CD, Quigley JG, Anderson SM, Malech HL, Abkowitz JL, Zanjani ED, Bodine DM: Improved transduction of human sheep repopulating cells by retrovirus vectors pseudotyped with feline leukemia virus type C or RD114 envelopes. Blood 2005, 106:51-58.

30. Kavanaugh MP, Miller DG, Zhang W, Law W, Kozak SL, Kabat D, Miller AD: Cell-surface receptors for gibbon ape leukemia virus and amphotropic murine retrovirus are inducible sodium-dependent phosphate symporters. Proc Natl Acad Sci USA 1994, 91:7071-7075.

31. O'Hara B, Johann SV, Klinger HP, Blair DG, Rubinson H, Dunne KJ, Sass P, Vitek SM, Robins T: Characterization of a human gene conferring sensitivity to infection by gibbon ape leukemia virus. Cell Growth Differ 1990, 1:119-127.

32. Tailor CS, Nouri A, Kabat D: A comprehensive approach to mapping the interacting surfaces of murine amphotropic and feline subgroup $B$ leukemia viruses with their cell surface receptors. J Virol 2000, 74:237-244.

33. Miller DG, Edwards RH, Miller AD: Cloning of the cellular receptor for amphotropic murine retroviruses reveals homology to that for gibbon ape leukemia virus. Proc Natl Acad Sci USA 1994, 91:78-82.

34. Eiden MV, Farrell KB, Wilson CA: Substitution of a single amino acid residue is sufficient to allow the human amphotropic murine leukemia virus receptor to function as a gibbon ape leukemia virus receptor. $J$ Virol 1996, 70:1080-1085.

35. Kurre P, Kiem H-P, Morris J, Heyward S, Battini J-L, Miller AD: Efficient transduction by an amphotropic retrovirus vector is dependent on highlevel expression of the cell surface virus receptor. J Virol 1999, 73:495-500.

36. Marchant D, Neil SJD, McKnight A: Human immunodeficiency virus types 1 and 2 have different replication kinetics in human primary macrophage culture. J Gen Virol 2006, 87:411-418.

37. Miller AD, Garcia JV, von Suhr N, Lynch CM, Wilson C, Eiden MV Construction and properties of retrovirus packaging cells based on gibbon ape leukemia virus. J Virol 1991, 65:2220-2224. 
38. Miller $A D$, Chen F: Retrovirus packaging cells based on 10A1 murine leukemia virus for production of vectors that use multiple receptors for cell entry. J Virol 1996, 70:5564-5571.

39. Bock M, Bishop KN, Towers G, Stoye JP: Use of a transient assay for studying the genetic determinants of Fv1 restriction. J Virol 2000, 74:7422-7430.

40. Marchant D, Neil SJ, Aubin K, Schmitz C, McKnight A: An envelopedetermined, $\mathrm{pH}$-independent endocytic route of viral entry determines the susceptibility of human immunodeficiency virus type 1 (HIV-1) and HIV-2 to Lv2 restriction. J Virol 2005, 79:9410-9418.

doi:10.1186/1742-4690-7-81

Cite this article as: Oliveira et al:: A novel envelope mediated post entry restriction of murine leukaemia virus in human cells is Ref1/TRIM5 $\alpha a$ independent. Retrovirology 2010 7:81.

\section{Submit your next manuscript to BioMed Central} and take full advantage of:

- Convenient online submission

- Thorough peer review

- No space constraints or color figure charges

- Immediate publication on acceptance

- Inclusion in PubMed, CAS, Scopus and Google Scholar

- Research which is freely available for redistribution

Submit your manuscript at www.biomedcentral.com/submit 